\title{
A Case Report of Possible Superfetation with Evidence of Ultrasound Findings, Gestational Age Calculations and Postnatal Complications
}

\author{
Taylor Alten, PA-S and Kevin Alten, MD* ii
}

Marietta College, USA

*Corresponding author: Kevin Alten, Marietta College, USA

\begin{abstract}
Introduction: Superfetation is a when second ovum is fertilized after there is already presence of an embryo in the uterine cavity. Human superfetation is extremely rare with less than 10 cases in the literature. According to previous research, the diagnosis of superfetation is suspected when there is a growth discrepancy in a multiple pregnancy.

Case presentation: We present a case of a 35-year-old gravid 1 para 0 who presented to her OB/GYN at 7 weeks by known last menstrual period (LMP) for her first prenatal visit. She was found to have single intrauterine pregnancy consistent with known LMP. At 12 weeks she had a cell free DNA (cfDNA) test performed that came back abnormal. The patient's next ultrasound at 19 weeks showed a twin gestation. Repeat cfDNA was normal for twin gestation and serial ultrasounds showed normal interval growth of both twins with the same discordance throughout the rest of the pregnancy. The cfDNA results, ultrasound findings demonstrating consistent growth discordance with normal interval growth otherwise, NICU management difference and placenta analysis post birth all support the diagnosis of superfetation.
\end{abstract}

Discussion: This case adds to the literature of human Superfetation.

\section{Case}

A 35-year-old gravid 1 para 0 presented to her $\mathrm{OB} / \mathrm{GYN}$ at 7 weeks by known last menstrual period (LMP) for her first prenatal visit. She has a history of hypertension and post-op deep vein thrombosis after an elective peroneal tendon repair. Her ultrasound that day demonstrated single intrauterine pregnancy consistent with her LMP (Figure 1A and Figure 1B). At
12 weeks, the patient had a cell free DNA (cfDNA) test performed that came back abnormal, the result being high-risk. Cell-free DNA is a serum-based screening test for aneuploidies using analysis of cell-free DNA fragments of placental trophoblasts that escape into the maternal circulation [1]. The report suggested this was due to either a vanishing twin, unrecognized multiple gestation or increased risk of fetal triploidy. No further ultrasounds were preformed until 19 weeks.

At 19 weeks and 2 days, the patient had a consult at a tertiary care facility with a maternal fetal medicine (MFM) physician for advanced maternal age and her high-risk cfDNA test. Ultrasound showed an undiagnosed twin gestation; Twin A measuring 18 weeks and 6 days and Twin B at 17 weeks and 2 days. The patient was advised to terminate the pregnancy due to fetal triploidy concerns for Twin B due to fetal growth restriction at such an early stage and her high-risk result cfDNA test. According to the patient, the possibility of her highrisk cfDNA being a result of her missed twin pregnancy diagnosis and not fetal triploidy was not discussed with her. She was offered amniocentesis but declined at that time.

2 weeks later, the patient sought a second opinion at another tertiary care facility. Ultrasound again demonstrated a twin pregnancy (Figure 2A and Figure 2B).

Measurements showed Twin A at 21 weeks and Twin $B$ at 19 weeks. The twins were both morphologically normal and had normal amniotic fluid measurements.

Citation: Alten T, Alten K (2021) A Case Report of Possible Superfetation with Evidence of Ultrasound Findings, Gestational Age Calculations and Postnatal Complications. Obstet Gynecol Cases Rev 8:202. doi.org/10.23937/2377-9004/1410202

Accepted: June 16, 2021: Published: June 18, 2021

Copyright: (C) 2021 Alten T, et al. This is an open-access article distributed under the terms of the Creative Commons Attribution License, which permits unrestricted use, distribution, and reproduction in any medium, provided the original author and source are credited. 


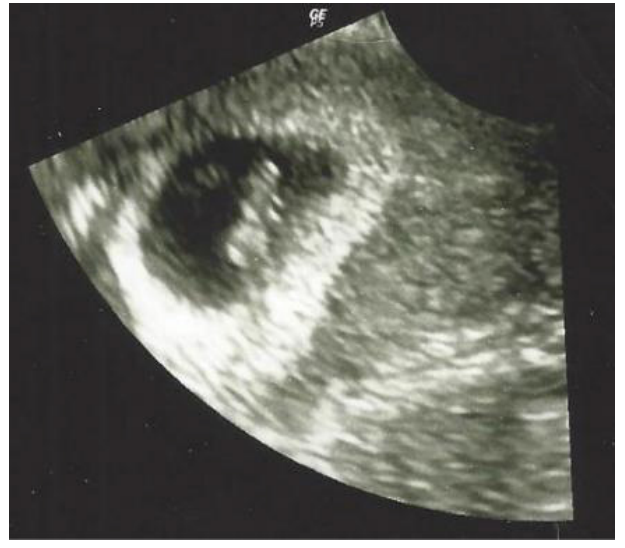

A

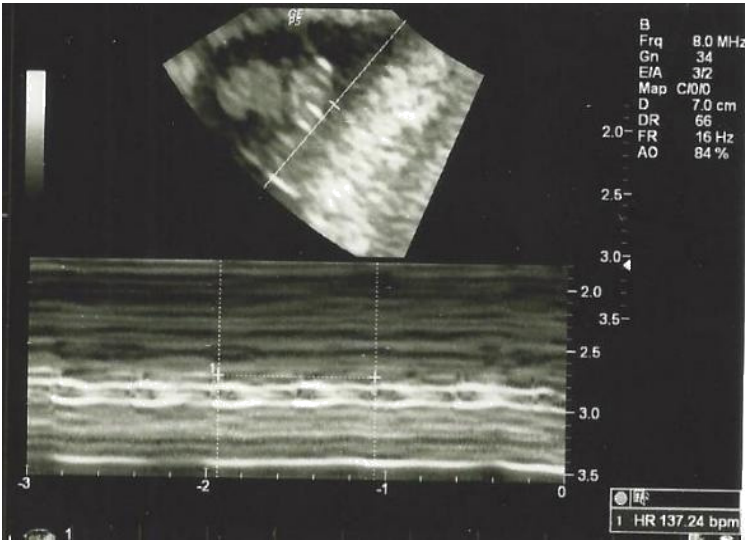

B

Figure 1: (A) 7 weeks, first ultrasound photo demonstrating one fetus present in the uterus; (B) 7 weeks, first ultrasound photo demonstrating fetal heart rate.

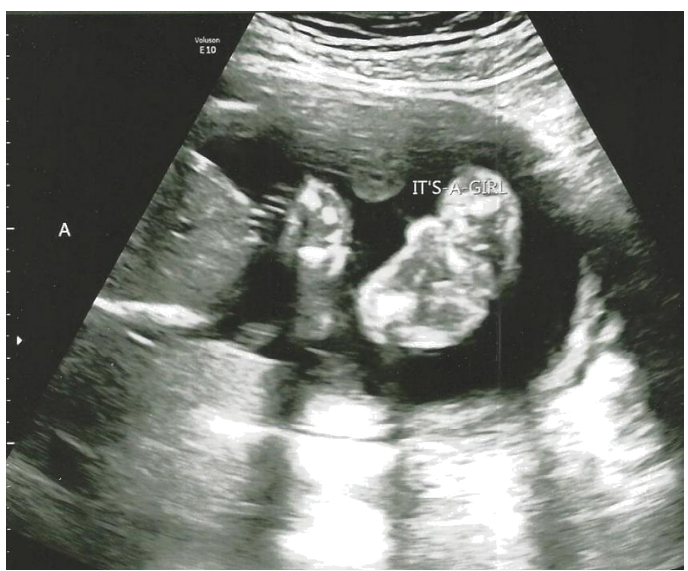

A

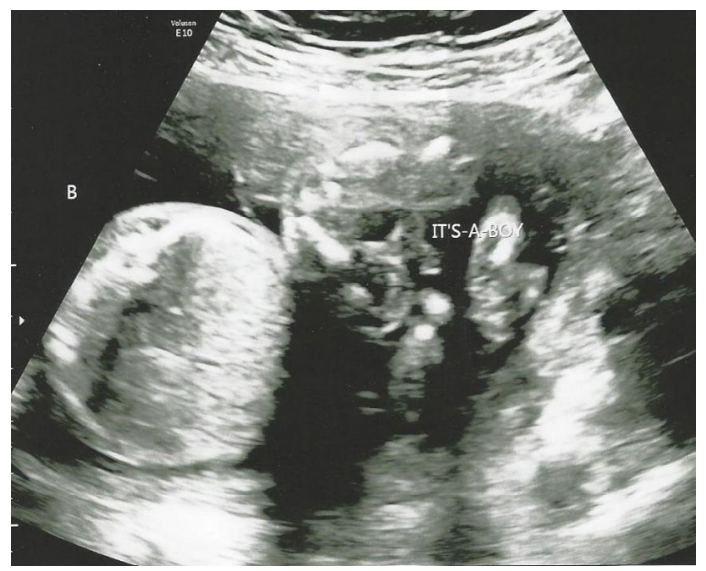

B

Figure 2: (A) Ultrasound photo showing Twin A, Female, measuring 21 weeks; (B) Ultrasound photo showing Twin B, Male, measuring 19 weeks.

These findings argued against fetal triploidy. Also, at this time, the initial cfDNA test was recalculated, accounting for the twin gestation, and another new cfDNA sample was drawn. The recalculated initial cfDNA result came back insufficient fetal DNA for diagnosis. The new cfDNA sample drawn at 21 weeks and 2 days result came back low-risk. The patient decided to continue with the pregnancy with close follow up. Serial ultrasounds were performed that showed normal interval growth of both twins with the same discordance as the first ultrasound at 19 weeks and 2 days (Graph 1 and Graph 2).

The patient was admitted to the hospital at 27 weeks and 5 days for daily fetal observation. Daily non-stress tests were performed and at 28 weeks and 3 days the twins were delivered by Caesarean section for nonreassuring fetal status.

Twin A was 965 grams, and a female and Twin B was 575 grams, and a male. Both were treated with surfactant at delivery.
Twin A neonatal intensive care (NICU) course was 160 days. She was on the ventilator 14 days. She was treated for reflux and feeding issues. The NICU doctor told the patient that, "her NICU course was like a normal 28-week baby".

Twin B NICU course was 192 days. He was on the ventilator for 42 days. He was more difficult to ventilate and more difficult to wean off the ventilator. The NICU doctor told the patient, "he is acting like a 25-week baby with his NICU course instead of a 28-week-old baby". At that time, the NICU team started to treat him like a 25week baby by slowing down ventilator weaning and giving him more time grow. When that change was made his NICU course was typical for a "25-week baby". He went home on 2 liters nasal cannula oxygen at night. He also had more feeding issues than Twin A and was discharged with a G-Tube for supplemental feedings at home.

\section{Discussion}

We feel this case demonstrates human superfetation. 


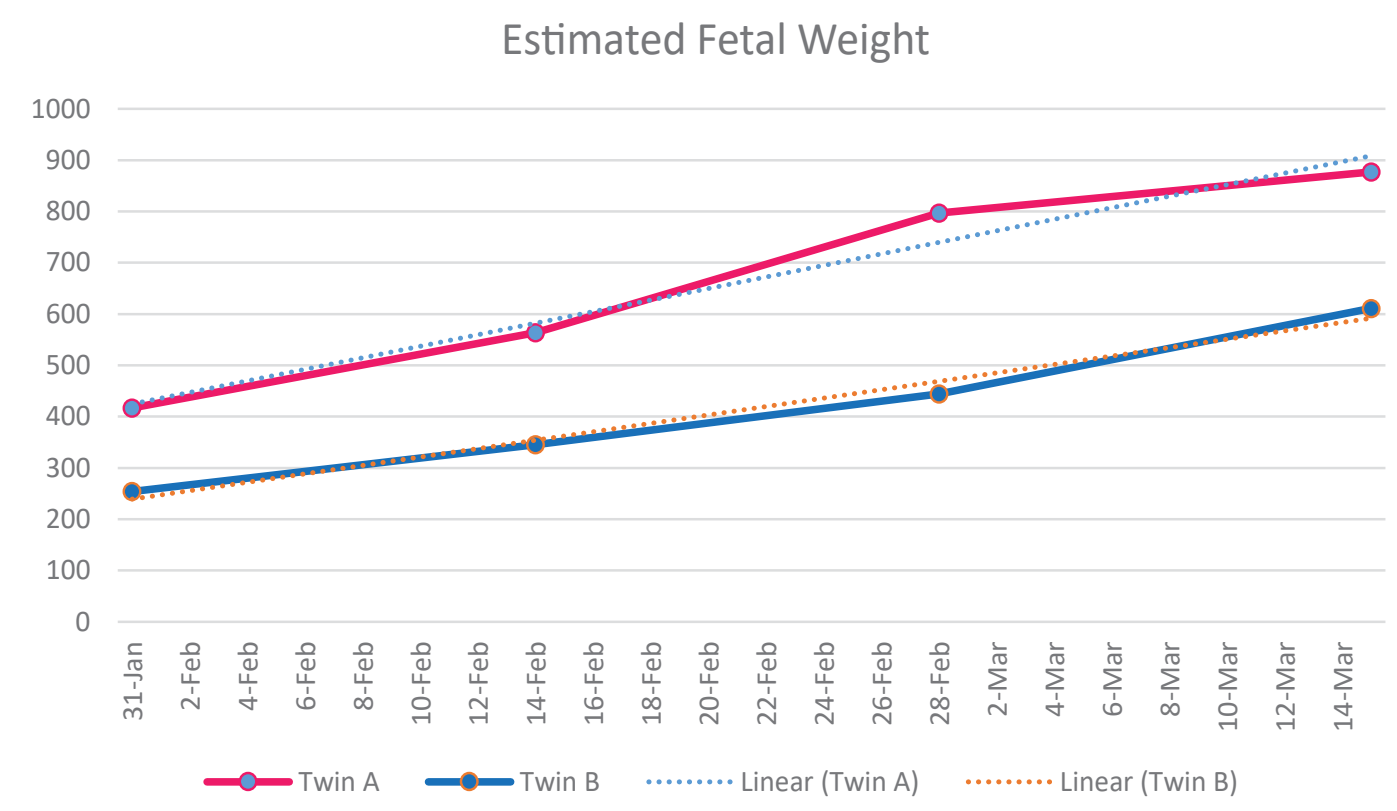

Graph 1: This graph shows the estimated fetal weight for Twin A and Twin B.

\section{\% Discordance}

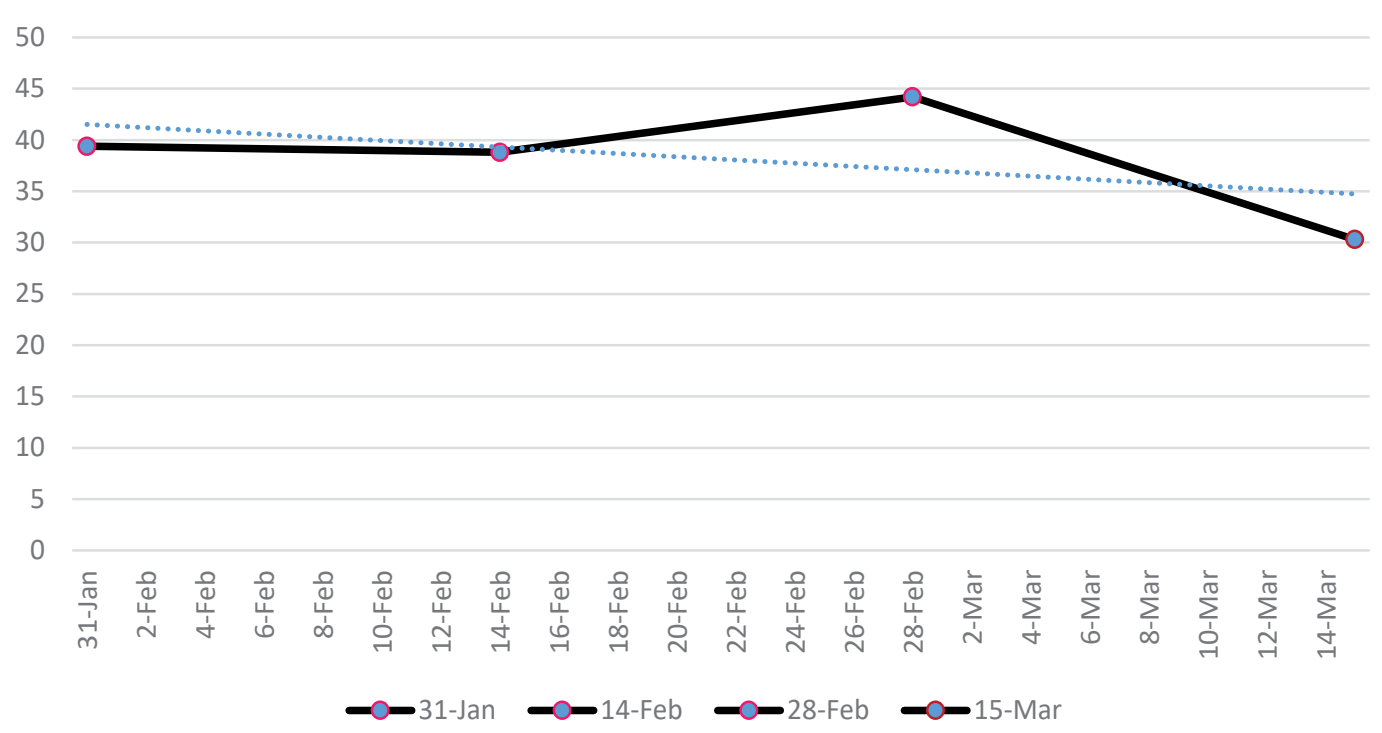

Graph 2: \% discordance from serial ultrasounds between Twin A and Twin B is shown above.

Superfetation is a rare phenomenon that occurs when a second ovum is fertilized after there is already presence of a fetus in the uterine cavity [2]. According to previous research, the diagnosis of superfetation is suspected when there is a growth discrepancy in a multiple pregnancy [3]. This is rare in humans with less than 10 cases of superfetation are described in the literature [4]. One of the early cases of superfetation was described in 1992 showing dichorionic diamniotic twins with a 4-week size and consistent growth difference shown through ultrasound measurements [5]. In this same case study at birth Twin 1 was 37 weeks and Twin 2 was 33-34 weeks [5]. In 1999, there was a similar case of superfetation where twins were born with a 4-week discordance that was confirmed throughout the pregnancy with ultrasound measurements [6]. In this case report dichorionic diamniotic twins were born with Twin 1 being 40 weeks and Twin 2 being 36 weeks [6]. Another case came from a personal story, a mom who had her first ultrasound at 7 weeks with one fetus seen in the uterus. A second ultrasound at 12 weeks showed a twin gestation [7]. She was told by her doctor that there was a 3-week size difference in the twins [7]. This continued throughout the entire pregnancy. The smaller child was tested for trisomy and results were normal [7]. The twins were born at 33 weeks with the boy measuring $4 \mathrm{lbs} 10 \mathrm{oz}$ and the girl measuring $2 \mathrm{lbs} 7 \mathrm{oz}$ [7]. She was told by her doctors that this was a possible 
case of superfetation [7]. This case has all the similar findings as our case. Specifically, the early ultrasound findings, gestational age calculations and postnatal complications that support our claim of superfetation.

As shown above in Figure $1 \mathrm{~A}$ and Figure $1 \mathrm{~B}$, it is seen that in the 7-week ultrasound only one fetus is seen in the uterine cavity. Normally when a woman is pregnant with twins you will see 2 fetuses present in the uterus at the 7-week ultrasound. It is extremely rare to miss twins at a 7-week ultrasound, especially when they are diamniotic dichorionic. In Figure $2 \mathrm{~A}$ and Figure 2B, it is seen at the 21-week ultrasound that 2 fetuses are shown in the uterine cavity for the first time. At this ultrasound growth discordance similar to the 19-week ultrasound was noted between Twin A and Twin B. Research has shown that initial cfDNA test fails (e.g., the results are not reported, indeterminate, uninterpretable, or nocall) have an increased risk of aneuploidy [8]. However, her first MFM consult did not discuss the possibility of a missed twin pregnancy diagnosis for this patient's initial high-risk cfDNA test. A missed superfetation twin pregnancy does explain her initial high-risk cfDNA result and the subsequent recalculated cfDNA being result of insufficient fetal DNA. The redrawn cfDNA test result of low-risk also points to two normal fetuses. On all of the subsequent ultrasounds that were done after 19 weeks there was always a consistent growth difference between the two fetuses but normal interval growth still occurred which is shown in Graph 1 . Since there was normal interval growth on subsequent ultrasounds it goes against the idea of fetal growth restriction due to triploidy and points more towards superfetation as the cause for growth differences [5]. The placenta examination post-operatively also points toward superfetation. The second placenta was much smaller and appeared to be "smashed" up against the other more normal appearing placenta. The NICU course of stay and subsequent management of Twin $B$ as being more premature than Twin A also points toward superfetation and not fetal growth restriction.

\section{Conclusion}

It is our belief that this is a case of superfetation.
The cfDNA results, ultrasound findings showing growth discordance with normal interval growth otherwise, NICU management difference and placenta analysis post birth point to evidence of superfetation. All the evidence points toward this conclusion and we feel this case adds to the literature of human superfetation.

\section{Key Points}

1. Superfetation is a when second ovum is fertilized after there is already presence of a fetus in the uterine cavity.

2. Human superfetation is extremely rare with less than 10 cases in the literature.

3. This case adds to the literature of human superfetation.

\section{References}

1. American College of Obstetricians and Gynecologists' Committee on Practice Bulletins - Obstetrics; Committee on Genetics; Society for Maternal-Fetal Medicine (2020) Screening for fetal chromosomal abnormalities: ACOG practice bulletin, Number 226. Obstet Gynecol 136: e48-e69.

2. Baijal N, Sahni M, Verma N, Kumar A, Parkhe N, et al. (2007) Discordant twins with the smaller baby appropriate for gestational age - unusual manifestation of superfoetation: A case report. BMC Pediatr 7: 2 .

3. Harrison A, Valenzuela A, Gardiner J, Sargent M, Chessex $P(2005)$ Superfetation as a cause of growth discordance in a multiple pregnancy. J Pediatr 147: 254-255.

4. Pape O, Winer N, Paumier A, Philippe HJ, FlatrEs B, et al. (2008) Superfetation: Case report and review of the literature. Journal De Gynecologie Obstetrique et Biologie de la Reporduction 37: 791-795.

5. Soudre G, Guettier X, Marpeau L, Larue L, Jault T, et al. (1992) In utero early suspicion of superfetation by ultrasound examination: A case report. Ultrasound Obstet Gynecol 2: 51-54.

6. Tuppen GD, Fairs C, de Chazal RC, Konje JC (1999) Spontaneous superfetation diagnosed in the first trimester with successful outcome. Ultrasound Obstet Gynecol 14: 219-221.

7. Rebecca Roberts Facebook page. This post is a personal account of superfetation.

8. Palomaki GE, Kloza EM (2018) Prenatal cell-free DNA screening test failures: A systematic review of failure rates, risks of down syndrome, and impact of repeat testing. Genet Med 20: 1312-1323. 